\title{
Curcumin May Enhance the Efficacy of Anti-Leukemia Drugs in Myeloid Leukemia
}

\author{
Apurba Mukherjee ${ }^{1}$, Kalyan Kusum Mukherjee ${ }^{2}$, \\ Sutapa Mukherjee ${ }^{1}$ and Madhumita $\operatorname{Roy}^{1^{*}}$ \\ ${ }^{1}$ Department of Environmental Carcinogenesis and Toxicology, Chittaranjan National Cancer \\ Institute, 37, S P Mukherjee Road, Kolkata - 700 026, India \\ ${ }^{2}$ Medical Oncology Department, Chittaranjan National Cancer Institute, 37, S P Mukherjee \\ Road, Kolkata - 700 026, India \\ *Corresponding author
}

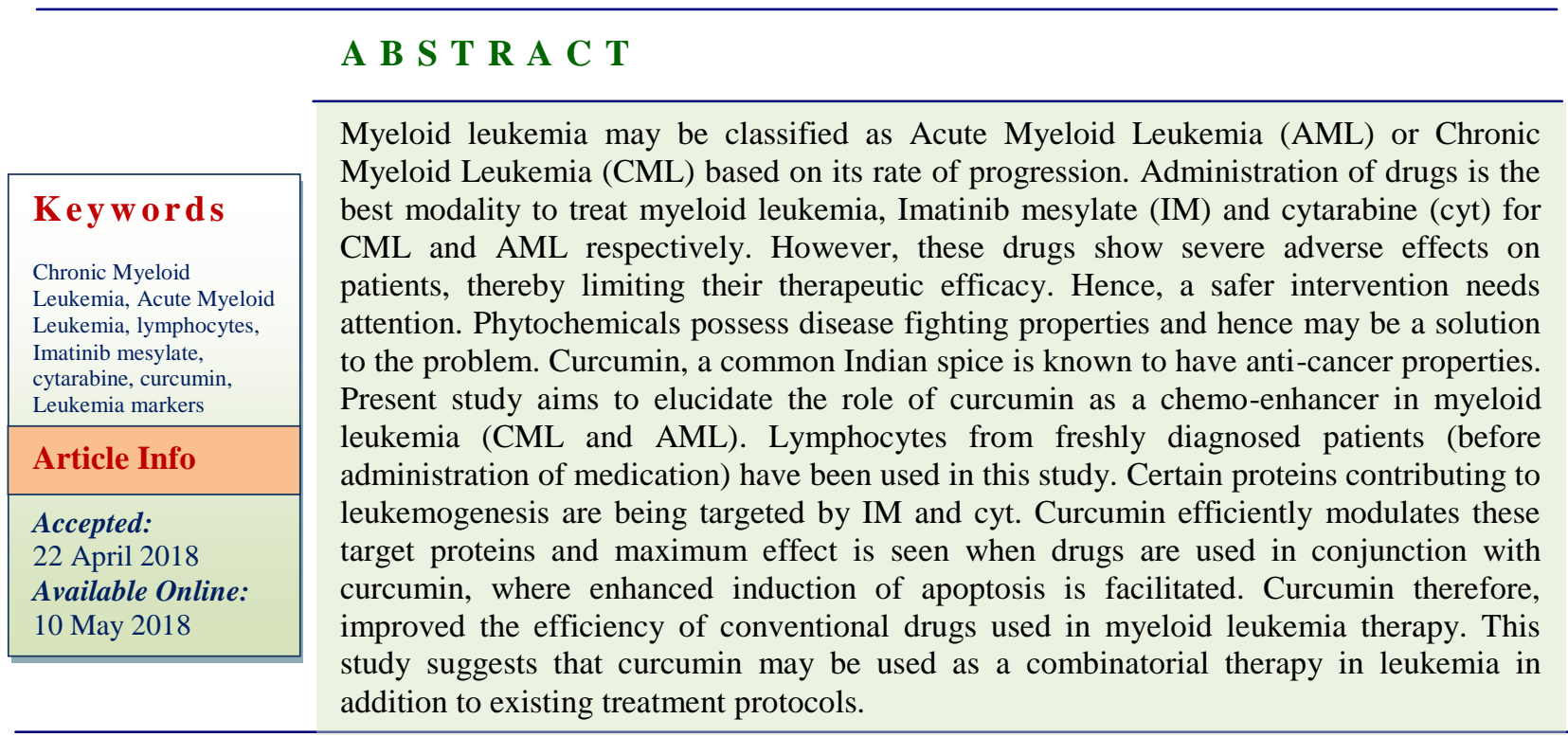

\section{Introduction}

Aberrant proliferation of myeloid progenitor cells in the bone marrow leads to myeloid leukemia, which may be classified into acute and chronic forms (Szczepański et al., 2003). Chronic myeloid leukemia (CML), the most common form of adult leukemia in India, is characterized by the reciprocal translocation between chromosomes 9 and 22, leading to a fusion Bcr-Abl gene, commonly known as Philadelphia chromosome (Machado et al., 2011). Acute myeloid leukemia (AML) on the other hand is a heterogeneous disease involving clonal proliferation of immature myeloid cells in the bone marrow and is one of the most lethal form of leukemia (Saultz and Garzon, 2016). Like all other cancers, various proteins viz., Tumor necrosis factoralpha (TNF- $\alpha$ ) and interleukins are aberrantly 
expressed, as a result of which signalling pathways like Janus kinase-Signal Transducer and Activator of Transcription (JAK-STAT), Mitogen-activated protein kinase (MAPK), and Phosphoinositide 3-kinase (PI3K/Akt) are anomalous. Deregulation of cytokines is associated with the etiology of myeloid leukemia (Kupsa et al., 2012). TNF- $\alpha$, a multifunctional cytokine and interleukins play pivotal role in leukemogenesis, owing to their involvement in inflammation and immunity (Goselink et al., 2000). Aberrant expressions of pro-inflammatory cytokines (IL-6 and IL-8) and anti-inflammatory cytokine IL-10 have been observed in myeloid leukemia patients (Sanchez-Correa et al., 2013). Abnormal regulation of signalling molecules like JAKSTAT, MAPK and PI3K/Akt leads to uncontrolled cell proliferation, survival, and thereby leukemogenesis. Binding of cytokines induces the JAK/STAT pathway and regulates various events in carcinogenesis. Constitutive activation of members of the STAT family, which normally require JAK-mediated phosphorylation for their transcriptional activation, has been observed in leukemia (Yamada and Kawauchi, 2013). MAP kinases are serine-threonine kinases that regulate malignant transformation and cell-cycle progression. Abnormal regulation of PI3K/Akt signalling network by cytokines plays pivotal role in the genesis of myeloid leukemia (Park et al., 2010). Chemotherapy/ targeted therapy continue to be the mainstay of treatment in myeloid leukemia. Imatinib mesylate (IM) is the drug of choice for CML, which acts by targeting the Philadelphia Chromosome (BcrAbl) (Sacha, 2014). The most acceptable drug for AML is Cytarabine (cyt), which acts by inhibition of DNA synthesis; it combines cytosine with arabinose instead of deoxyribose, thereby causing damage to DNA (Löwenberg et al., 2011). Though IM and cyt are effective in tackling the disease, they are bestowed with severe adverse side effects as they target not only the leukemia cells but also the healthy cells. Hence natural compounds (phytochemicals) having antitumor activities (Roy et al., 2015a) and minimal toxicity (Sarkar et al., 2014a; Mukherjee et al., 2015) in combination with anti-neoplastic drugs may be explored for effective treatment of leukemia. The phytochemicals have a unique feature that they show preferential effect on cancer cells only; Indian spice Curcumin, having a plethora of medicinal value may be a good option in this regard.

Present study is an ex-vivo one conducted on lymphocytes isolated from CML and AML patients. Lymphocytes from healthy donors were used as control for the study. This study aims to determine whether a combinatorial therapy of curcumin and conventional antileukemia drugs will be an effective strategy in leukemia so that the drug dose and associated toxicity may be minimized.

\section{Materials and Methods}

\section{Materials}

Cell culture media RPMI-1640 and Phytohaemagglutinin (PHA) were purchased from GIBCO-BRL India Pvt. Ltd, New Delhi, India. N, N'-methylenebisacrylamide, Acrylamide, Fetal Bovine Serum (FBS) and kits for ELISA were obtained from Invitrogen BioServices India Pvt. Ltd., Bangalore, India. Histopaque, bovine serum albumin (BSA), dithiothreitol (DTT), Ponceau S, 4-(2hydroxyethyl)-1-piperazineethanesulfonic acid (HEPES), CHAPS, RNase A, proteinase K, ethylene glycol-O,-O'-bis,(2-aminoethyl) N,N,N',N'-tetra acetic acid (EGTA), propidium iodide (PI), curcumin and IM were purchased from Sigma, MO, USA. Nitrocellulose membrane was purchased from Hybond ECL, Amersham Biosciences, UK. Tris, glycine, 5-bromo-4-chloro-3-indolyl phosphate/nitro blue tetrazolium (BCIP-NBT) and sodium dodecyl sulfate (SDS) were 
obtained from Amresco, Ohio, USA. Goat anti-rabbit IgG-alkaline phosphatase conjugate was obtained from Abcam. Activities of caspases 3, 8 and 9 were determined using assay kits from Millipore, Billerica, MA. Antibodies against TNF- $\alpha$ (Biorbyt); IL-8 and IL-10 (Abcam); p38 Map Kinase, Ras, Raf, PI3K, Akt, JAK2, STAT3 (Genetex) were used for western blotting purpose. Anti-BCRABL Monoclonal Antibody [7C6] (MA1-153) was procured from Pierce, Thermo Scientific, USA. Other reagents were of analytical grade and were purchased locally.

\section{Methods}

\section{Selection of patients}

The study was conducted on AML and CML patients visiting Chittaranjan National Cancer Institute. Necessary permission from Institutional Ethics Committee was taken; vide IEC Number A-4.311/39/2014, where the informed consent form, inclusion and exclusion criteria were approved. Freshly diagnosed 50 patients in the age group of 1860 years were recruited in each group, who are yet to receive any treatment. Patients were diagnosed by the doctors based on patient history, clinical examination, bone marrow examination and complete blood count. For CML patients, only Bcr-Abl positive cases were taken, based on FISH analysis done in a reputed diagnostic laboratory. Healthy individuals, who are non-smokers, not having any chronic disease were taken as study controls.

\section{Isolation and culture of lymphocytes}

This is an ex-vivo study. Heparinized blood was layered on top of Histopaque and centrifuged to get a buffy coat rich in lymphocytes. The isolated lymphocytes were seeded in RPMI-1640 media supplemented with $10 \%$ FBS, antibiotics and $20 \mu \mathrm{g} / \mathrm{ml}$
Phytohaemagglutinin (PHA). The cells were cultured at $37^{\circ} \mathrm{C}$ in a humidified atmosphere of $5 \% \mathrm{CO}_{2} / 95 \%$ air.

\section{Treatment protocol}

Lymphocytes were treated with drugs $(3.6 \mu \mathrm{M}$ IM and $84.0 \mu \mathrm{M}$ cyt) alone, as well as in presence of Curcumin $(30 \mu \mathrm{M})$ for 24 hours. These doses of drugs were chosen based on the $\mathrm{EC}_{50}$ values (concentration causing 50\% cell killing), as obtained from MTT assay.

\section{Assessment of cell cytotoxicity}

Cytotoxicity of antileukemic drugs to lymphocytes was measured by MTT assay, which is based on the principle that mitochondrial dehydrogenase enzyme reduces 3-(4, 5-dimethythiazol-2-yl)-2,5-diphenyl tetrazolium bromide (MTT) in metabolically active cells; activity level being directly proportional to cell viability. The MTTformazan product dissolved in DMSO gives a purple colour, which was estimated spectrophotometrically at $570 \mathrm{~nm}$.

\section{Enzyme-linked immunosorbent assay}

The cytokine levels (TNF- $\alpha$, IL-6, IL-8, and IL-10) were estimated using ELISA kits as described previously (Mukherjee et al., 2016).

\section{Western blotting analysis}

Expressions of Bcr-Abl, p38 MAPK, Ras, Raf, PI3K, Akt, JAK2, STAT3 were examined using Western blot (WB) analysis technique using appropriate antibodies (Sarkar et al., 2013). $\beta$-actin was used as loading control.

\section{RNA extraction and semi quantitative RT PCR}

RNAqueous 4PCR kit (Ambion) was used to extract total RNA from cells following 
laboratory protocol and Polymerase Chain Reaction was performed (Roy et al., 2015b).

\section{Assessment of apoptosis by TUNEL assay}

Induction of programmed cell death was studied using in situ Apoptosis Detection Kit as per prescribed protocol. Harvested cells were fixed with $4 \%$ paraformaldehyde $(\mathrm{pH}$ 7.4). Methanol containing $0.3 \% \quad \mathrm{H}_{2} \mathrm{O}_{2}$ was used to remove endogenous peroxidases, followed by addition of permeabilization buffer. Cells undergoing apoptosis were labelled with fluorescein-dUTP. Anti-FITC HRP Conjugate was then added and slides were incubated followed by addition of DAB. The cells were counterstained with methyl green. The apoptotic cells were observed under light microscope.

\section{Assessment of caspase activity}

Cell lysates were prepared to measure the activities of caspases 3,8 and 9 following manufacturer's protocol (Mukherjee et al., 2016).

\section{Statistical analysis}

Statistical analysis was done using SPSS 10.0 (one way ANOVA followed by Dunett t-test).

\section{Results and Discussion}

\section{Pilot study}

A pilot study has been conducted with 10 freshly diagnosed myeloid leukemia patients in each group. Peripheral blood lymphocytes (PBL) have been isolated from healthy human donors (control) and the patients who were free from metastatic and locally recurrent disease. Western blot has been conducted to assess the expression of several tumor markers (TNF- $\alpha$, IL-6, IL-8, IL-10, p38 MAPK, Ras, Raf, PI3K, Akt, JAK2, STAT3). These proteins are expressed at much higher levels in leukemia patients as compared to normal healthy individuals, except IL-10, whose expression is just the reverse, though there is inter-individual variation. The results are shown in Figure 1; Western blot bands in Figure $1 \mathrm{~A}$ and the corresponding band intensities in Figure 1B. The results are consistent with our previous finding with leukemia cell lines. MTT assay was conducted to estimate the toxicity of IM and cyt. It has been observed that increasing doses of both IM and cyt induce killing of lymphocytes isolated from myeloid leukemia patients (Figure 1C). The $\mathrm{EC}_{50}$ values of IM and cyt has been calculated to be $3.6 \mu \mathrm{M}$ and $84.0 \mu \mathrm{M}$ respectively. Upto a concentration of $50 \mu \mathrm{M}$, curcumin is non-toxic in lymphocytes from healthy donors, whereas beyond $30 \mu \mathrm{M}$ concentration it was reported that extent of killing of leukemia cells is quite high (Mukherjee et al., 2016); hence for further studies in order to obtain substantial number of cells to work with, the concentration of curcumin was set at $30 \mu \mathrm{M}$.

Further, it has been studied whether curcumin increases the efficacy of these anti-leukemia drugs towards cell killing. Lymphocytes from leukemia patients have been treated with different concentrations of drugs along with increasing doses of curcumin for 24 hours. The doses selected for IM are 0.5, 1, 2.5 and $5 \mu \mathrm{M}$ and that for cyt are $0.1,1,10$ and 100 $\mu \mathrm{M}$. It has been seen that curcumin increases the cytotoxicity of the drugs (Figure 1C). The $\mathrm{EC}_{50}$ values have been calculated and are shown in Table 1. Curcumin increases the relative efficacy of IM and cyt by 1.89 and 2.43 respectively.

Based on these findings, 40 more patients have been selected in each group and further experiments have been conducted to prove the fact that curcumin may act as an enhancer of anti-leukemia drugs. 


\section{Modulation of leukemia markers}

The levels of cytokines have been assessed with the lymphocytes isolated from leukemia patients, treated with drugs and/or curcumin. Pro-inflammatory cytokines (TNF- $\alpha$, IL-6 and IL-8) are down-regulated by drugs; curcumin enhances the effect. IL-10, an antiinflammatory cytokine on the other hand, is unaffected by IM, but cyt positively influences IL-10 levels. Curcumin, when added along with the drugs, increases the levels of IL-10 in both the cases (Figure 2). It has been observed from Western blot results that Curcumin, IM and cyt could individually reduce the expression of these proteins in lymphocytes isolated from CML and AML patients (Figure $3 \mathrm{~A})$. As evident from the bands, the extent of down-regulation is more when the drugs and curcumin are used in conjunction. The corresponding band intensities are represented in the bar diagrams (Figure 3B).

Fig.1 Preliminary study to assess constitutive expression of tumor markers and drug dose. (A). Constitutive expressions of various proteins implicated in myeloid leukemia were assessed by western blot in lymphocytes isolated from CML patients (I), AML patients (II) and normal healthy donor (III). $\beta$-actin was used as loading control. (B). Corresponding band intensities representing average of 10 individuals in each group \pm SE are depicted as bar diagrams. (C). Lymphocytes from CML and AML patients were treated with $\operatorname{IM}(0.5,1,2.5,5 \mu \mathrm{M})$ and cyt $(0.1,1,10,100 \mu \mathrm{M})$ respectively in absence and presence of curcumin $(0,0.5,1,10,30 \mu \mathrm{M})$ for 24 hours. Curcumin increases the cytotoxicity of the drugs. Values are significant ${ }^{\mathrm{a}}(\mathrm{p}<0.01)$, ${ }^{b}(p<0.005),{ }^{c}(p<0.0005)$ with respect to cells treated with drugs alone

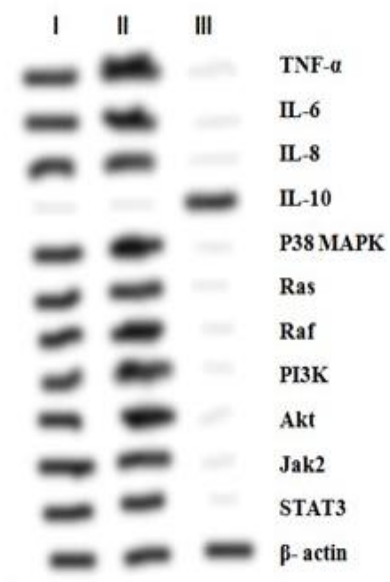

A

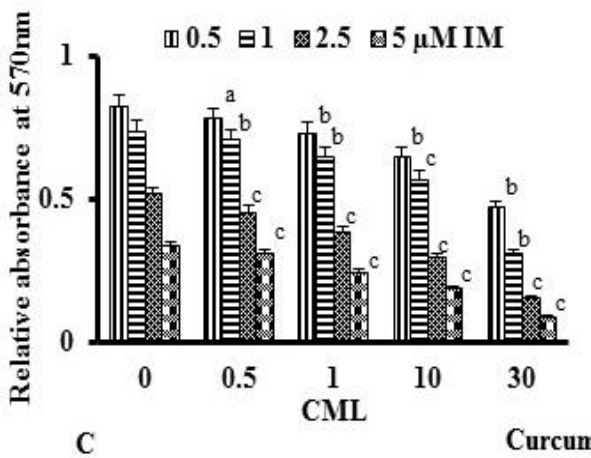

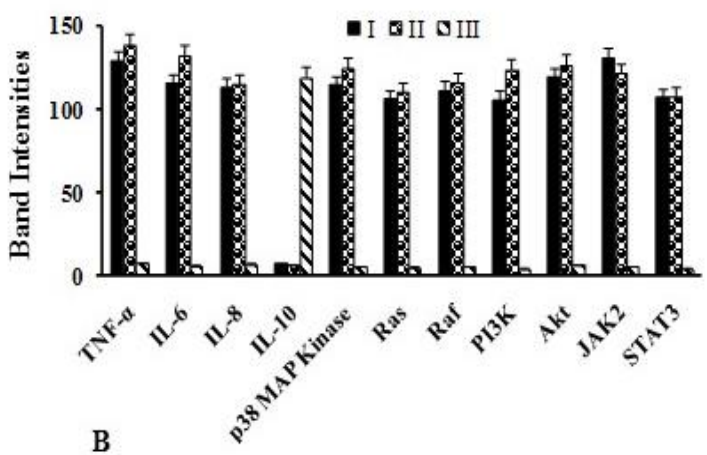


Fig.2 Effect of drugs on cytokine levels as influenced by curcumin. Lymphocytes isolated from CML and AML patients were treated with drugs $(3.6 \mu \mathrm{M}$ IM and $84.0 \mu \mathrm{M}$ cyt respectively) and/or curcumin $(30 \mu \mathrm{M})$ for 24 hours. The levels of cytokines were assessed by ELISA technique. Cytokine levels were found to be regulated by drugs; however, the extent of modulation was much more in presence of curcumin. Values are average of 40 patients in each group \pm SE and are significant ${ }^{\mathrm{a}}(\mathrm{p}<0.01),{ }^{\mathrm{b}}(\mathrm{p}<0.005),{ }^{\mathrm{c}}(\mathrm{p}<0.0005)$ with respect to untreated cells
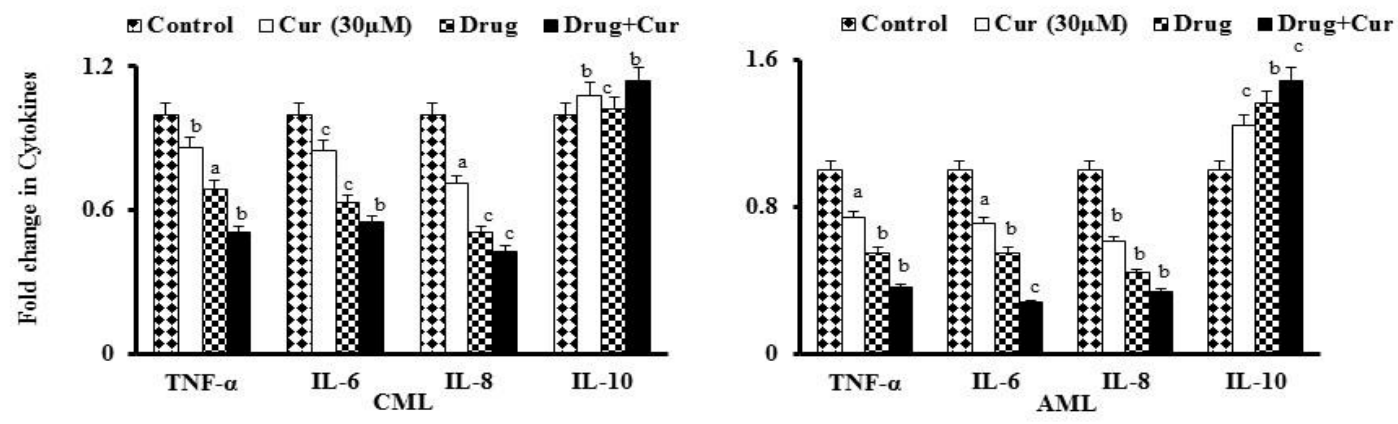

Fig.3 Effect of drugs on expression of proteins involved in leukemogenesis. (A). Western blotting was used to assess the expression of tumor markers in lymphocytes after treating the cells with drugs $(3.6 \mu \mathrm{M}$ IM for CML and $84.0 \mu \mathrm{M}$ cyt for AML) alone and in combination with $30 \mu \mathrm{M}$ curcumin. Lane 1 represents control cells, Lane 2 represents curcumin treated cells, Lane 3 represents cells treated with drugs, Lane 4 represents cells treated with both curcumin and drugs. Maximum modulation of markers has been observed in the combinatorial treatment modality. (B). Corresponding band intensities, which are average of 40 individuals $\pm \mathrm{SE}$ in each group are depicted in the bar diagrams. (C). Modulation of expression of Bcr-Abl, a characteristic feature of CML was assessed at the genetic level by RT-PCR. (D). Western blot bands show modulation of Bcr-Abl at the protein level by IM and/or curcumin. (E).

Corresponding band intensities have been depicted as bar diagram. Decrease in Bcr-abl was much higher when IM and curcumin was used in conjunction. The results are significant ${ }^{\mathrm{a}}(\mathrm{p}<0.01),{ }^{\mathrm{b}}(\mathrm{p}<0.005),{ }^{\mathrm{c}}(\mathrm{p}<0.0005)$ with respect to untreated cells

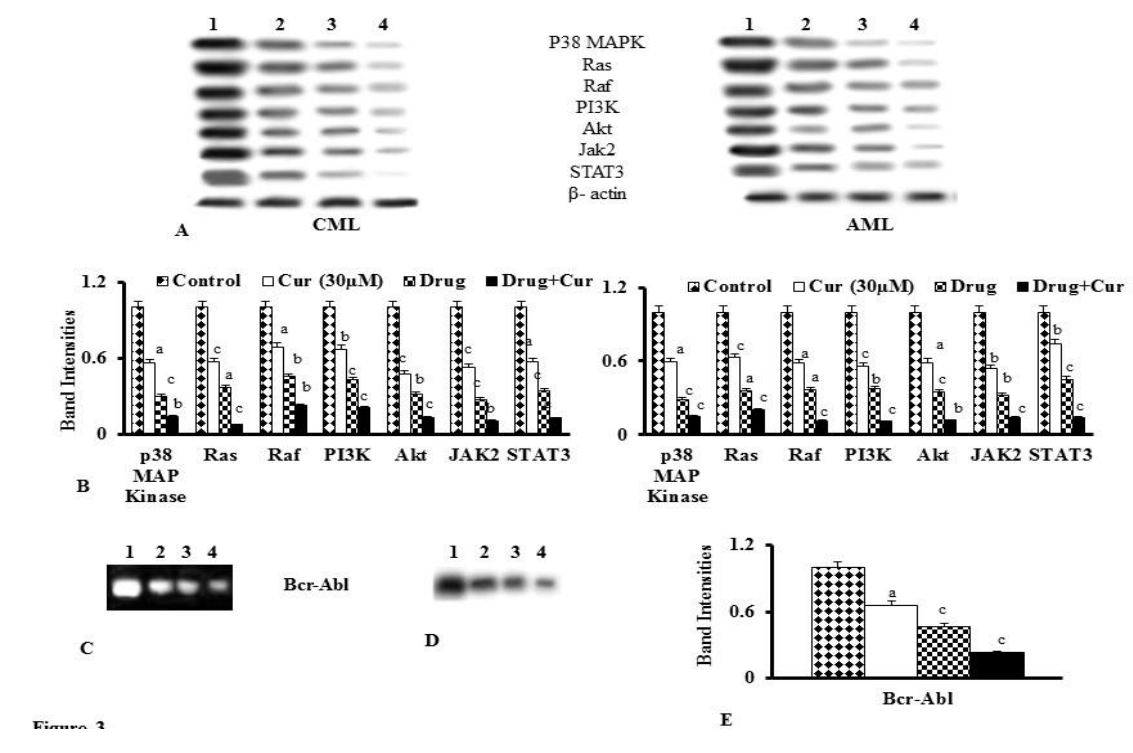


Fig.4 Induction of apoptosis by anti-leukemia drugs as influenced by curcumin. (A). Activation of caspases 3, 8 \& 9 was observed in peripheral blood lymphocytes (PBL) after treatment of cells with drugs (3.6 $\mu \mathrm{M}$ IM for CML and $84.0 \mu \mathrm{M}$ cyt for AML) and/or curcumin. Activation of caspase 9 was more than caspases 3 and 8, thereby suggesting that intrinsic pathway of apoptosis

is involved. (B). Representative micrographs showing induction of apoptosis as revealed by

TUNEL assay results. (1) represents control cells, (2) represents curcumin treated cells, (3) represents cells treated with drugs, (4) represents cells treated with both curcumin and drugs. (C).

Based on these results, apoptotic index was calculated and are depicted in the bar diagram.

Results show that programmed cell death facilitated by drugs gets enhanced in presence of curcumin. Values represent mean of 40 patients in each group \pm SE and are significant ${ }^{\mathrm{a}}(\mathrm{p}<0.01)$,

${ }^{b}(p<0.005),{ }^{c}(p<0.0005)$ with respect to untreated cells
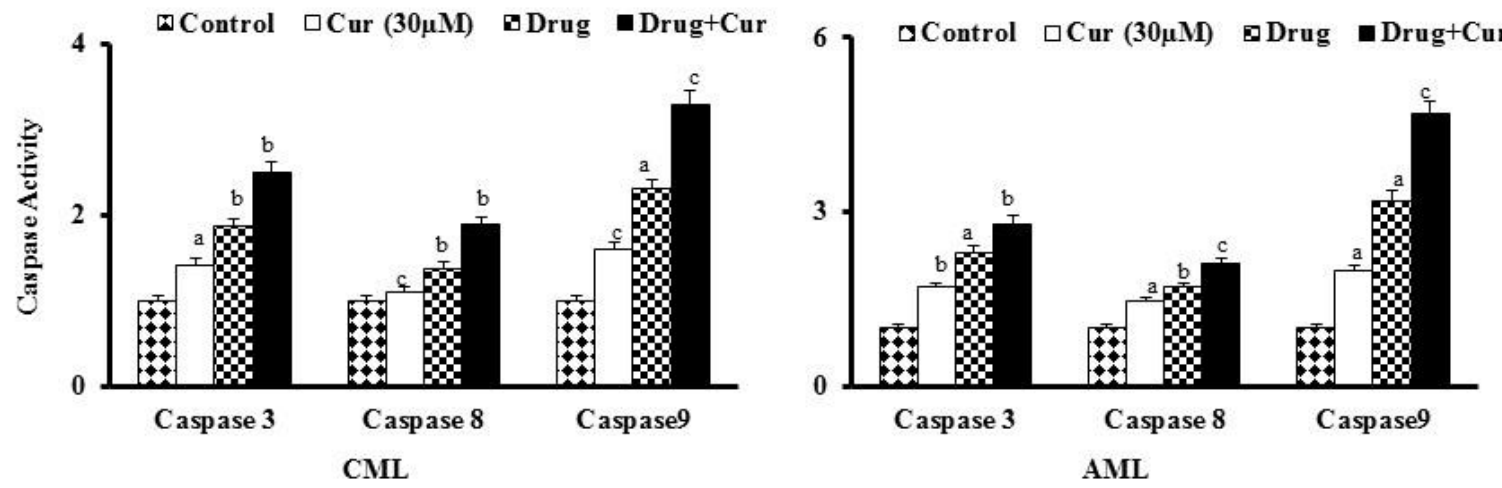

A

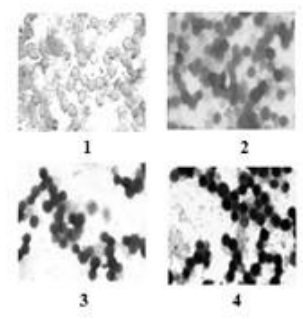

CML

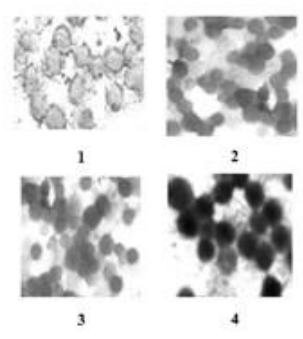

AML

B

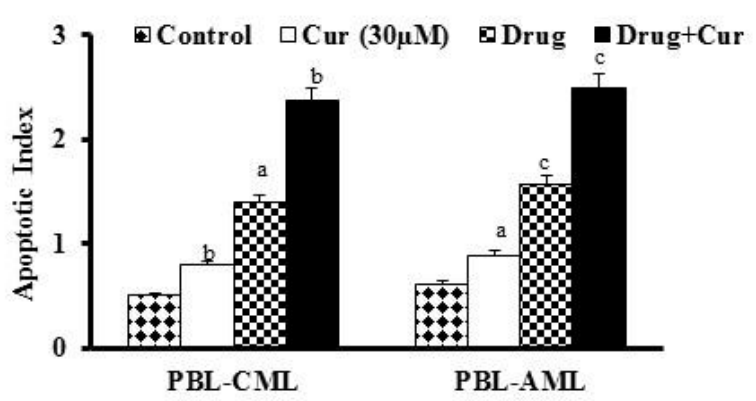

C

Table.1 Curcumin increases the efficacy of anti-leukemia drugs Imatinib Mesylate and cytarabine

\begin{tabular}{|l|l|l|l|l|}
\hline Cell & Drug & Compound & $\mathrm{EC}_{50}(\boldsymbol{\mu M})$ & Relative Efficacy \\
\hline PBL-CMIL & IM & Cur- & 3.6 & 1.0 \\
\cline { 3 - 4 } & \multirow{2}{*}{ Cyt } & Cur+ & 1.9 & 1.89 \\
\hline & PBL-AMI & Cur- & 84 & 1.0 \\
\cline { 3 - 3 } & Cur+ & 34.56 & 2.43 \\
\hline
\end{tabular}


Bcr-Abl is a characteristic hallmark of CML. The level of this marker has been assessed in lymphocytes of CML patients by RT-PCR at the genetic level (Figure 3C) and by western blot at the protein level (Figure 3D). IM reduces the expression of Bcr-Abl both at the protein and genetic level. However, the effect is more pronounced in presence of curcumin. The corresponding band intensities are depicted in Figure 3E.

\section{Activation of caspases}

Caspases are critical regulators of the apoptotic event and activities of caspases 3,8 and 9 have been determined. Results show that both IM and cyt induce activation of caspases in treated lymphocytes and curcumin enhances the effect prominently (Figure 4A). It is evident from the graphs that the increase in activities of caspase 3 and caspase 9 are more than that of caspase 8 , thereby indicating that intrinsic pathway is responsible for induction of apoptosis in this case.

\section{Induction of apoptosis}

TUNEL assay was employed to ascertain induction of apoptosis in lymphocytes isolated from leukemia patients. Apoptotic cells have been viewed under a light microscope and representative micrographs are shown in Figure 4B. The number of apoptotic cells has been counted and apoptotic index (i.e. the ratio of the apoptotic to the non-apoptotic cells) has been calculated. The values are depicted in the bar diagram (Figure 4C).

Despite rapid advancements in understanding the biology of myeloid leukemia and development of new therapeutic strategies, the disease, especially acute type continues to have poor prognosis. The main barrier to successful remission is the toxic effects (Krug et al., 2011) associated with existing therapeutic regimens (chemotherapeutics and targeted therapy). Imatinib mesylate and cytarabine are the mostly commonly used drugs for CML and AML respectively, both focusing on various signalling molecules involved in leukemogenesis. However apart from leukemia cells, these drugs, affect other healthy cells as well. Therefore, though effective in treating the disease, they pose serious adverse effects on the health of patients. Non-toxic means of leukemia control thus need to be warranted. Plant derived molecules or phytochemicals are often blessed with anti-cancer properties.

They also act differentially on cancer cells, sparing the normal ones (Biswas et al., 2015). Thus inclusion of these phytochemicals in the therapeutic regimen of cancer may help to combat the problem of adverse toxicity of existing therapeutics. Curcumin, a commonly used Indian spice, is one such example.

It has been reported earlier from the Department of Environmental Carcinogenesis and Toxicology, CNCI that curcumin is non-toxic to lymphocytes isolated from normal healthy individuals upto the dose tested but it is toxic to cancer cells (Mukherjee et al., 2016).

Previous studies from our laboratory have shown that curcumin helps to increase the efficacy of IM and cyt in leukemia cell lines K562 and HL-60 respectively by modulation of various important markers and induction of apoptosis (Sarkar et al., 2014b). It has also been shown that curcumin helps to reduce drug dose.

The present work is an ex vivo study, conducted with lymphocytes isolated from CML and AML patients (50 patients in each group) to elucidate the potential of curcumin as a combinatorial therapy in myeloid leukemia. Lymphocytes isolated were treated with anti-leukemia drugs (IM or cyt) and curcumin, either alone or in combination. A pilot study was conducted with lymphocytes from 10 patients in each group. It was found that leukemia markers TNF- $\alpha$, IL-6, IL-8, IL-10, p38 MAPK, Ras, Raf, PI3K, Akt, JAK2, STAT3 are aberrantly expressed in leukemia cells as compared to normal controls. The results are consistent with findings from other laboratories (Hemmati et al., 2017). 
Cytotoxicity of IM and cyt to lymphocytes of patients were tested and the $\mathrm{EC}_{50}$ values were calculated. It was found that when curcumin was administered to cells along with the drugs, the $\mathrm{EC}_{50}$ values of the drugs were dampened. Thus, the efficacies of the drugs were found to increase in presence of curcumin as the cells were sensitive to lower concentration of drugs in presence of the phytochemical.

Based on these results, further experiments were conducted to elucidate the underlying mechanisms. It was observed that IM and cyt could modulate aberrant expression of leukemic markers but the effect was much more when curcumin was present along with the drugs. In the combinatorial therapy, modulation of these markers enhances programme cell death, as revealed by the apoptotic index values and increase in caspase activity.

Thus, results of this preliminary study suggest that curcumin may help to improve the efficiency of IM and cyt, thereby reducing drug dose and associated toxicity. An indication of the potential of curcumin is indicated, but being an ex vivo work, nothing can be concluded concretely. Further clinical studies are warranted, where curcumin will be given directly to the patients along with the drugs to establish its potential as an adjuvant therapy in myeloid leukemia.

\section{Acknowledgement}

Authors are indebted to DST-INSPIRE for partial funding and to Director, CNCI for providing necessary infrastructure. Thanks are due to Institutional Ethics Committee for providing necessary permission to carry out this study.

\section{References}

Biswas, J., M. Roy and Mukherjee, A. 2015. Anticancer Drug Development Based on Phytochemicals. J Drug Discov Develop and Deliv. 2(1): 1012.

Goselink, H.M., R. Willemze, and Falkenburg, J.H.F. 2000. Tumor necrosis factor alpha
(Tnf- $\alpha$ ) Production by acute myeloid leukemic (Aml) Blasts results in impaired proliferation of normal hematopoietic progenitor cells $(\mathrm{Hpc})$. Experimental Hematology. 28(7): 69-70.

Hemmati, S., T. Haque, and Gritsman, K. 2017. Inflammatory Signaling Pathways in Preleukemic and Leukemic Stem Cells. Front Oncol. 7: 265.

Krug, U., T. Büchner, W.E. Berdel, and C. Müller-Tidow. 2011. The Treatment of Elderly Patients with Acute Myeloid Leukemia. Dtsch Arztebl Int. 108(51-52): 863-870.

Kupsa, T., J.M. Horacek, and Jebavy, L. 2012. The role of cytokines in acute myeloid leukemia: a systematic review. Biomed Pap Med Fac Univ Palacky Olomouc Czech Repub. 156(4): 291-301.

Löwenberg, B., T. Pabst, E.Vellenga, W. van Putten, H.C. Schouten, C. Graux, A. Ferrant, P. Sonneveld, B.J, Biemond, A. Gratwohl, G.E. de Greef, L.F. Verdonck, M.R. Schaafsma, M. Gregor, M. Theobald, U. Schanz, J. Maertens, and Ossenkoppele G.J., Dutch-Belgian Cooperative Trial Group for HematoOncology (HOVON) and Swiss Group for Clinical Cancer Research (SAKK) Collaborative Group. 2011. Cytarabine dose for acute myeloid leukemia. N Engl J Med. 364(11): 1027-1036.

Machado, M.P., J.P. Tomaz, I. LorandMetze, C.A.de Souza, A.C. Vigorito, M. T. Delamain, I. Bendit, N.F. Pereira, and Pagnano, K.B.B. 2011. Monitoring of BCR-ABL levels in chronic myeloid leukemia patients treated with imatinib in the chronic phase - the importance of a major molecular response. Rev Bras Hematol Hemoter. 33(3): 211-215.

Mukherjee, A., R. Sarkar, S. Mukherjee, J. Biswas and Roy, M. 2016. Curcumin Boosts up the Efficacy of Imatinib Mesylate in Chronic Myelogenic Leukemia Cell Line K-562 by Modulation of Various Markers. Int.J.Curr.Microbiol.App.Sci. 5(12): 240255 . 
Mukherjee, A., S. Mukherjee, J. Biswas and Roy, M. 2015. Phytochemicals in obesity control. Int.J.Curr.Microbiol. App.Sci. 4(4): 558-567.

Park, S., N. Chapuis, J. Tamburini, V. Bardet, P. Cornillet-Lefebvre, L. Willems, A. Green, P. Mayeux, C. Lacombe, and Bouscary, D.2010. Role of the PI3K/AKT and mTOR signaling pathways in acute myeloid leukemia. Haematologica. 95(5): 819-828.

Roy M, R. Sarkar, A. Mukherjee, and Mukherjee, S. 2015b. Inhibition of crosstalk between Bcr-Abl and PKC signaling by PEITC, augments imatinib sensitivity in chronic myelogenous leukemia cells. Chem. Biol. Interact, 242, 195-201.

Roy, M., A.B. Kunnumakkara, A. Mukherjee, R. Sarkar, S. Mukherjee and Biswas, J. 2015a. Repair Activity Impaired by Arsenic: Recovery by Phytochemicals Int.J.Curr.Microbiol.App.Sci. 4(3): 578587.

Sacha, T. 2014. Imatinib in Chronic Myeloid Leukemia: an Overview. Mediterr J Hematol Infect Dis. 6(1): e2014007.

Sanchez-Correa, B., J.M. Bergua, C. Campos, I. Gayoso, M.J. Arcos, H. Bañas, S. Morgado, J.G. Casado, R. Solana, and Tarazona, R. 2013. Cytokine profiles in acute myeloid leukemia patients at diagnosis: survival is inversely correlated with IL-6 and directly correlated with IL10 levels. Cytokine. 61(3): 885-91.

Sarkar, R., A. Mukherjee, R. Biswas, J. Biswas and Roy, M. 2014a. Sulphoraphane, by virtue of its antioxidant potential downregulates HSP90 in leukemia cells Int.J.Curr. Microbiol.App.Sci. 3(1): 476486.

Sarkar, R., A. Mukherjee, S. Mukherjee, R. Biswas, J. Biswas, and Roy, M. 2014b. Curcumin augments the efficacy of antitumor drugs used in leukemia by modulation of heat shock proteins via HDAC6. J. Environ. Pathol. Toxicol. Oncol, 33(3), 247-263.

Sarkar, R., S. Mukherjee, and Roy, M. 2013. Targeting heat shock proteins by phenethyl isothiocyanate results in cellcycle arrest and apoptosis of human breast cancer cells. Nutr. Cancer, 65(3), 480-493.

Saultz, J.N. and Garzon R. 2016. Acute Myeloid Leukemia: A Concise Review. J Clin Med. 5(3): 33.

Szczepański, T., V.H. van der Velden and van Dongen, J.J. 2003. Classification systems for acute and chronic leukaemias. Best Pract Res Clin Haematol. 16(4): 561-582.

Yamada, O. and Kawauchi, K.2013. The role of the JAK-STAT pathway and related signal cascades in telomerase activation during the development of hematologic malignancies. JAKSTAT. 2(4): e25256.

\section{How to cite this article:}

Apurba Mukherjee, Kalyan Kusum Mukherjee, Sutapa Mukherjee and Madhumita Roy. 2018. Curcumin May Enhance the Efficacy of Anti-Leukemia Drugs in Myeloid Leukemia. Int.J.Curr.Microbiol.App.Sci. 7(05): 3274-3283. doi: https://doi.org/10.20546/ijcmas.2018.705.383 